Ciência e Natura, Santa Maria

v.40, Edição Especial: II mostra de Projetos da UFSM -

Campus Cachoeira do Sul, 2018, p. 183-191

Revista do Centro de Ciências Naturais e Exatas - UFSM

ISSN impressa: 0100-8307 ISSN on-line: 2179-460X

\title{
Conexões entre grafos e matrizes na modelagem de problemas matemáticos
}

Connections between graphs and matrices in modeling mathematical problems

Larissa Melchiors Furlan ${ }^{1}$, Mylena Roehrs ${ }^{2}$ e Glauber Rodrigues de Quadros $^{3}$

1,2,3 Universidade Federal de Santa Maria - Campus Cachoeira do Sul, Cachoeira do Sul, Brasil larimelfurlan@hotmail.com; mylenaroehrs00@hotmail.com; glauber.quadros@ufsm.br

\section{Resumo}

A teoria dos grafos é muito importante no mundo matemático como uma excelente forma de conexão com o mundo real. Utilizando-se a teoria de grafos dirigidos é possivel transformar muitos dos problemas cotidianos em problemas matemáticos, de forma a fazer um estudo exato em cada caso. Neste trabalho são exploradas as matrizes relacionadas aos diversos tipos de grafos, como a matriz de vértices, a qual é associada a um grafo dirigido, e a matriz de adjacência. Mais do que isso, são construídas matrizes de conexões de vários passos, de forma a criar múltiplas conexões entre os vértices de um grafo dirigido. Em seguida, colocaremos diversas aplicações destes resultados na forma de exemplos.

Palavras-chave: Grafos; Teoria de grafos; Matriz de vértices

\section{Abstract}

Graphs theory is very important in the mathematical world as an excellent way of connecting with the real world. By using the theory of directed graphs it is possible to transform many of the everyday problems into mathematical problems, so as to make an exact study in each case. In this work we explore the matrices related to the various types of graphs, such as the vertex matrix, which is associated with a directed graph, and the adjacency matrix. Moreover, matrices of multi-step connections are constructed so as to separate the various blades between the vertices of a directed graph. Then, we will construct some applications of those results in the form of examples.

Keywords: Graphs; Graphs theory; Vertex matrix 


\section{Introdução}

A teoria de grafos é muito útil e importante no mundo matemático, por possibilitar fácil visualização das conexões entre pontos desejados. Através dos grafos, pode-se transformar problemas do cotidiano em problemas matemáticos, possibilitando assim sua análise. Um grafo é composto por conjuntos ordenados de vértices e as arestas que podem ou não ligá-los. (COSTA, 2011). Por exemplo, a hierarquia da cadeia alimentar pode ser expressa em grafos, onde os vértices seriam os animais que compõe a cadeia e as arestas a relação alimentar, como um animal que come o outro. Então é possível analisar matematicamente a cadeia alimentar, elaborando as matrizes e assim identificando quem afeta quem neste grupo observado. Um grafo pode ter pontos isolados e para este trabalho, será considerado que não terá um vértice ligado nele mesmo sem passar por outro e também pode ter partes desconexas. As arestas que ligam os vértices podem ser representadas por arcos ou retas, com setas indicando a relação dos pontos, como 'A' ligado a 'B', e se não houver, nesse caso, ligação de 'B' para 'A', a seta terá único sentido. A cada grafo pode ser atribuída uma matriz de vértices, na qual quando um vértice for ligado no outro recebe como entrada 1 e caso contrário, 0 . Um vértice pode ligar a outro através de diferentes "caminhos", formando então conexões de 1 ou mais passos, que é quando um ponto está ligado a outro indiretamente, sendo feito pelos outros pontos. Por exemplo, um ponto 'A' pode ser ligado a um ponto 'C' através de um ponto 'B', portanto é uma conexão de 2 passos. A estas novas conexões de mais passos podem ser relacionadas novas matrizes, multiplicando a original de um passo por ela mesma a quantidade de vezes desejada. Por exemplo, a matriz das conexões de 2 passos será a original ao quadrado.

Este trabalho não tem o intuito de apresentar novos elementos à teoria dos grafos, por isso a mesma é inserida de forma simples, gradual e direta. Na verdade, com este trabalho, pretende-se apenas apresentar a teoria de grafos, utilizando exemplos criados de forma a refletir problemas cotidianos, podendo então fazer a análise matemática permitida pela transformação do problema em um grafo.

\section{Desenvolvimento teórico}

\subsection{Grafos}

Um grafo é um conjunto de pontos ordenados $\left(\mathrm{P}_{1}, \mathrm{P}_{2}, \mathrm{P}_{3}, \ldots, \mathrm{P}_{\mathrm{n}}\right)$, chamados vértices, que podem ou não estar ligados entre si por "flechas", denominadas arcos ou arestas. Quando um vértice está ligado a outro, utiliza-se a notação $P_{i} \rightarrow P_{j}$, que é lido como " $P_{i}$ está conectado a $P_{j}$ ”. Ao mesmo tempo em que $P_{i}$ está conectado a $P_{j}$, o contrário também pode ser verdadeiro, denotando-se então $P_{i} \leftrightarrow P_{j}$. (ANTON, H.; RORRES, C., 2012).

Como exemplo, pode-se citar o seguinte grafo:

Figura 1 - exemplo de grafo.

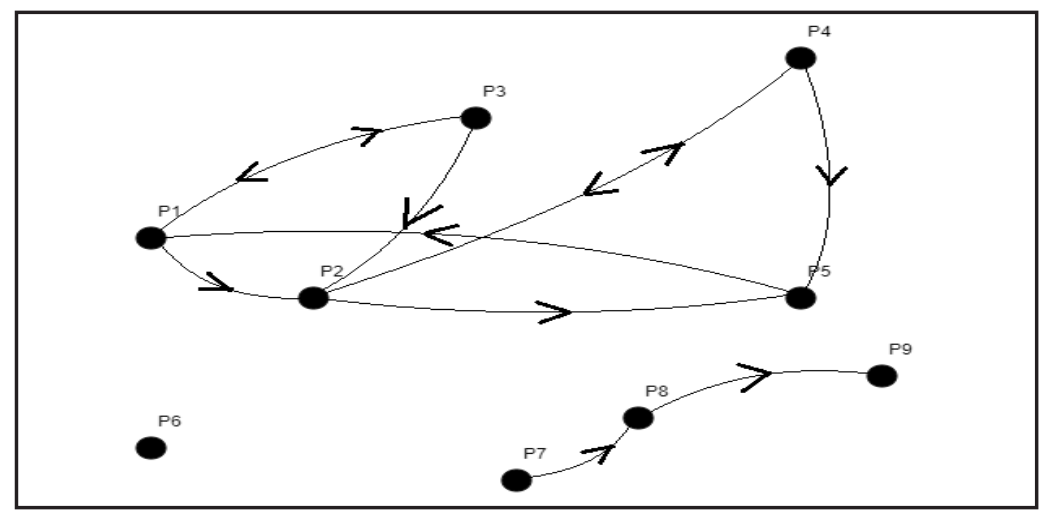

Fonte: própria autora 


\subsection{Matriz de vértices}

A todo grafo pode ser atribuído uma matriz de vértices $M$ de tamanho $\mathrm{n} \times \mathrm{n}$ que por definição recebe 1 na entrada $\mathrm{m}_{\mathrm{ij}}$ quando $\mathrm{P}_{\mathrm{i}} \rightarrow \mathrm{P}_{\mathrm{j}}$ e 0 caso contrário. Como não nos é interessante que um vértice seja ligado a ele mesmo, a diagonal principal será sempre 0 (ANTON, H.; RORRES, C., 2012).

No caso, a matriz do grafo anterior é:

$$
M=\left[\begin{array}{lllllllll}
0 & 1 & 1 & 0 & 0 & 0 & 0 & 0 & 0 \\
0 & 0 & 0 & 1 & 1 & 0 & 0 & 0 & 0 \\
1 & 1 & 0 & 0 & 0 & 0 & 0 & 0 & 0 \\
0 & 1 & 0 & 0 & 1 & 0 & 0 & 0 & 0 \\
1 & 0 & 0 & 0 & 0 & 0 & 0 & 0 & 0 \\
0 & 0 & 0 & 0 & 0 & 0 & 0 & 0 & 0 \\
0 & 0 & 0 & 0 & 0 & 0 & 0 & 1 & 0 \\
0 & 0 & 0 & 0 & 0 & 0 & 0 & 0 & 1 \\
0 & 0 & 0 & 0 & 0 & 0 & 0 & 0 & 0
\end{array}\right]
$$

É possível analisar através da matriz as conexões existentes entres os vértices, sendo que a linha indica o vértice analisado e a coluna o vértice ao qual ele está ligado. Por exemplo, na linha 1 é observado o vértice P1, que está ligado ao P2 e ao P3, já que as entradas nestas colunas são 1 . Onde as entradas são 0 indica que P1 não está ligado ao vértice correspondente ao número da coluna, por exemplo, P1 não está ligado a P4.

\subsection{Matrizes de conexões de mais de um passo}

Um ponto não se liga a outro por apenas um caminho, ou seja, por uma única conexão de 1 passo. Existem outras opções que podem levar um ponto a outro, ditas conexões de 2 (ou mais) passos, variando o número de vértices pelo qual se "passa" para chegar de um ponto a outro. A matriz de 2 passos equivale a matriz de vértices elevada ao quadrado e representa as conexões que passam por 1 vértice da origem ao destino $\left(\mathrm{P}_{\mathrm{i}} \rightarrow \mathrm{P}_{\mathrm{j}} \rightarrow \mathrm{P}_{\mathrm{k}}\right)$. A matriz de 3 passos equivale a matriz de vértices elevada ao cubo, a matriz de 4 passos equivale a matriz de vértices elevada à quarta e assim sucessivamente. As entradas das matrizes de mais passos são o número de conexões com aquele determinado número de passos que existe de um vértice a outro. (ANTON, H.; RORRES, C., 2012).

Como exemplo, é feita a matriz de 2 passos do grafo anterior:

$$
\mathrm{M}^{2}=\left[\begin{array}{lllllllll}
1 & 1 & 0 & 1 & 1 & 0 & 0 & 0 & 0 \\
1 & 1 & 0 & 0 & 1 & 0 & 0 & 0 & 0 \\
0 & 1 & 1 & 1 & 1 & 0 & 0 & 0 & 0 \\
1 & 0 & 0 & 1 & 1 & 0 & 0 & 0 & 0 \\
0 & 1 & 1 & 0 & 0 & 0 & 0 & 0 & 0 \\
0 & 0 & 0 & 0 & 0 & 0 & 0 & 0 & 0 \\
0 & 0 & 0 & 0 & 0 & 0 & 0 & 0 & 0 \\
0 & 0 & 0 & 0 & 0 & 0 & 0 & 0 & 0 \\
0 & 0 & 0 & 0 & 0 & 0 & 0 & 0 & 0
\end{array}\right]
$$


É possível ver com esta matriz outras possibilidades de conexão, sendo conexões de 2 passos, e o número destas conexões existentes entre um vértice e outro. Por exemplo, P1 pode ser ligado a ele mesmo através do P3, o que denota na entrada $\mathrm{m}_{11}$ uma conexão de dois passos. Têm-se as seguintes conexões possíveis:

$$
\begin{array}{|l|l|l|l|l|}
\hline \mathrm{P} 1 \rightarrow \mathrm{P} 3 \rightarrow \mathrm{P} 1 & \mathrm{P} 2 \rightarrow \mathrm{P} 4 \rightarrow \mathrm{P} 2 & \mathrm{P} 3 \rightarrow \mathrm{P} 2 \rightarrow \mathrm{P} & \mathrm{P} 4 \rightarrow \mathrm{P} 2 \rightarrow \mathrm{P} 4 & \\
\mathrm{P} 1 \rightarrow \mathrm{P} 3 \rightarrow \mathrm{P} 2 & \mathrm{P} 2 \rightarrow \mathrm{P} 5 \rightarrow \mathrm{P} 1 & \mathrm{P} \rightarrow \mathrm{P} 1 \rightarrow \mathrm{P} 3 & \mathrm{P} 4 \rightarrow \mathrm{P} 2 \rightarrow \mathrm{P} 5 & \mathrm{P} 1 \rightarrow \mathrm{P} 2 \\
\mathrm{P} 1 \rightarrow \mathrm{P} 2 \rightarrow \mathrm{P} 4 & \mathrm{P} 2 \rightarrow \mathrm{P} 4 \rightarrow \mathrm{P} 5 & \mathrm{P} 1 \rightarrow \mathrm{P} 2 & \mathrm{P} 4 \rightarrow \mathrm{P} 5 \rightarrow \mathrm{P} 1 & \\
\mathrm{P} 1 \rightarrow \mathrm{P} 2 \rightarrow \mathrm{P} 5 & & \mathrm{P} 1 \rightarrow \mathrm{P} 2 \rightarrow \mathrm{P} 4 & \\
\hline
\end{array}
$$

E ainda, é possível fazer a matriz de três passos deste mesmo grafo:

$$
\mathrm{M}^{3}=\left[\begin{array}{lllllllll}
1 & 2 & 1 & 1 & 2 & 0 & 0 & 0 & 0 \\
1 & 1 & 1 & 1 & 1 & 0 & 0 & 0 & 0 \\
2 & 2 & 0 & 1 & 2 & 0 & 0 & 0 & 0 \\
1 & 2 & 1 & 0 & 1 & 0 & 0 & 0 & 0 \\
1 & 1 & 0 & 1 & 1 & 0 & 0 & 0 & 0 \\
0 & 0 & 0 & 0 & 0 & 0 & 0 & 0 & 0 \\
0 & 0 & 0 & 0 & 0 & 0 & 0 & 0 & 0 \\
0 & 0 & 0 & 0 & 0 & 0 & 0 & 0 & 0 \\
0 & 0 & 0 & 0 & 0 & 0 & 0 & 0 & 0
\end{array}\right]
$$

Com esta matriz, pode-se observar ainda mais opções de caminho e vértices que possuem mais de um caminho entre si, como é o caso da entrada $\mathrm{m}_{12}$ que é 2, ou seja, tem duas possibilidades de caminho de 3 passos de P1 para P2. Existem as seguintes possíveis conexões:

\begin{tabular}{|l|l|l|l|l|}
\hline $\mathrm{P} 1 \rightarrow \mathrm{P} 2 \rightarrow \mathrm{P} 5 \rightarrow \mathrm{P} 1$ & $\mathrm{P} 3 \rightarrow \mathrm{P} 1 \rightarrow \mathrm{P} 3 \rightarrow \mathrm{P} 1$ & & \\
$\mathrm{P} 1 \rightarrow \mathrm{P} 2 \rightarrow \mathrm{P} 4 \rightarrow \mathrm{P} 2$ & $\mathrm{P} 2 \rightarrow \mathrm{P} 4 \rightarrow \mathrm{P} 5 \rightarrow \mathrm{P} 1$ & $\mathrm{P} 3 \rightarrow \mathrm{P} 1 \rightarrow \mathrm{P} 2 \rightarrow \mathrm{P} 5$ & $\mathrm{P} 4 \rightarrow \mathrm{P} 2 \rightarrow \mathrm{P} 4 \rightarrow \mathrm{P} 2$ & $\mathrm{P} 5 \rightarrow \mathrm{P} 1 \rightarrow \mathrm{P} 3 \rightarrow \mathrm{P} 1$ \\
$\mathrm{P} 1 \rightarrow \mathrm{P} 3 \rightarrow \mathrm{P} 2 \rightarrow \mathrm{P} 4$ & $\mathrm{P} 2 \rightarrow \mathrm{P} 5 \rightarrow \mathrm{P} 1 \rightarrow \mathrm{P} 2$ & $\mathrm{P} 3 \rightarrow \mathrm{P} 1 \rightarrow \mathrm{P} 2 \rightarrow \mathrm{P} 4$ & $\mathrm{P} 4 \rightarrow \mathrm{P} 2 \rightarrow \mathrm{P} 5 \rightarrow \mathrm{P} 1$ & $\mathrm{P} 5 \rightarrow \mathrm{P} 1 \rightarrow \mathrm{P} 3 \rightarrow \mathrm{P} 2$ \\
$\mathrm{P} 1 \rightarrow \mathrm{P} 3 \rightarrow \mathrm{P} 2 \rightarrow \mathrm{P} 5$ & $\mathrm{P} 2 \rightarrow \mathrm{P} 5 \rightarrow \mathrm{P} 1 \rightarrow \mathrm{P} 3$ & $\mathrm{P} 3 \rightarrow \mathrm{P} 2 \rightarrow \mathrm{P} 5 \rightarrow \mathrm{P} 1$ & $\mathrm{P} 4 \rightarrow \mathrm{P} 5 \rightarrow \mathrm{P} 1 \rightarrow \mathrm{P} 2$ & $\mathrm{P} 5 \rightarrow \mathrm{P} 1 \rightarrow \mathrm{P} 2 \rightarrow \mathrm{P} 4$ \\
$\mathrm{P} 1 \rightarrow \mathrm{P} 2 \rightarrow \mathrm{P} 4 \rightarrow \mathrm{P} 5$ & $\mathrm{P} 2 \rightarrow \mathrm{P} 4 \rightarrow \mathrm{P} 2 \rightarrow \mathrm{P} 4$ & $\mathrm{P} 3 \rightarrow \mathrm{P} 2 \rightarrow \mathrm{P} 4 \rightarrow \mathrm{P} 2$ & $\mathrm{P} 4 \rightarrow \mathrm{P} 5 \rightarrow \mathrm{P} 1 \rightarrow \mathrm{P} 3$ & $\mathrm{P} 5 \rightarrow \mathrm{P} 1 \rightarrow \mathrm{P} 2 \rightarrow \mathrm{P} 5$ \\
$\mathrm{P} 1 \rightarrow \mathrm{P} 3 \rightarrow \mathrm{P} 1 \rightarrow \mathrm{P} 2$ & $\mathrm{P} 2 \rightarrow \mathrm{P} 4 \rightarrow \mathrm{P} 2 \rightarrow \mathrm{P} 5$ & $\mathrm{P} 3 \rightarrow \mathrm{P} 2 \rightarrow \mathrm{P} 4 \rightarrow \mathrm{P} 5$ & $\mathrm{P} 4 \rightarrow \mathrm{P} 2 \rightarrow \mathrm{P} 4 \rightarrow \mathrm{P} 5$ & \\
$\mathrm{P} 1 \rightarrow \mathrm{P} 3 \rightarrow \mathrm{P} 1 \rightarrow \mathrm{P} 3$ & & $\mathrm{P} 3 \rightarrow \mathrm{P} 1 \rightarrow \mathrm{P} 3 \rightarrow \mathrm{P} 2$ & & \\
\hline
\end{tabular}

\subsection{Grafo dirigido por dominância}

Em muitos grupos existe uma hierarquia, uma ordem de dominação bem direcionada entre quaisquer dois membros de determinado grupo. Dados dois indivíduos A e B, ou A domina B ou B domina A, mas nunca ambos. Em termos de grafos dirigidos, quando $P_{i} \rightarrow P_{j}$, significa que $P_{i}$ domina $P_{j}$. No mundo animal pode-se observar esta relação claramente em um sistema onde exista presa e predador, o predador dominará a presa, porém a presa não será capaz de dominar o predador. (ANTON, H.; RORRES, C., 2012). 
Um grafo dirigido por dominância é um grafo tal que dado qualquer par de vértices distintos $\mathrm{P}_{\mathrm{i}}$ e $\mathrm{P}_{\mathrm{j}}$, ou $\mathrm{P}_{\mathrm{i}}$ dominará $\mathrm{P}_{\mathrm{j}}\left(\mathrm{P}_{\mathrm{i}} \rightarrow \mathrm{P}_{\mathrm{j}}\right)$ ou $\mathrm{P}_{\mathrm{j}}$ dominará $\mathrm{P}_{\mathrm{i}}\left(\mathrm{P}_{\mathrm{j}} \rightarrow \mathrm{P}_{\mathrm{i}}\right)$, mas não será encontrado um caso em que um domine o outro simultaneamente $\left(\mathrm{P}_{\mathrm{i}} \leftrightarrow \mathrm{P}_{\mathrm{j}}\right)$. (ANTON, H.; RORRES, C., 2012).

Exemplo: Em uma teia alimentar composta por lobos, raposas, águias, lebres, cervos e plantas, os cervos e as lebres se alimentam das plantas, os lobos comem os cervos e as raposas, as águias atacam as lebres e as raposas e as raposas comem as lebres. É possível montar um grafo com esta teia alimentar para analisá-la:

Figura 2 - exemplo de grafo dirigido por dominância.

Fonte: própria autora

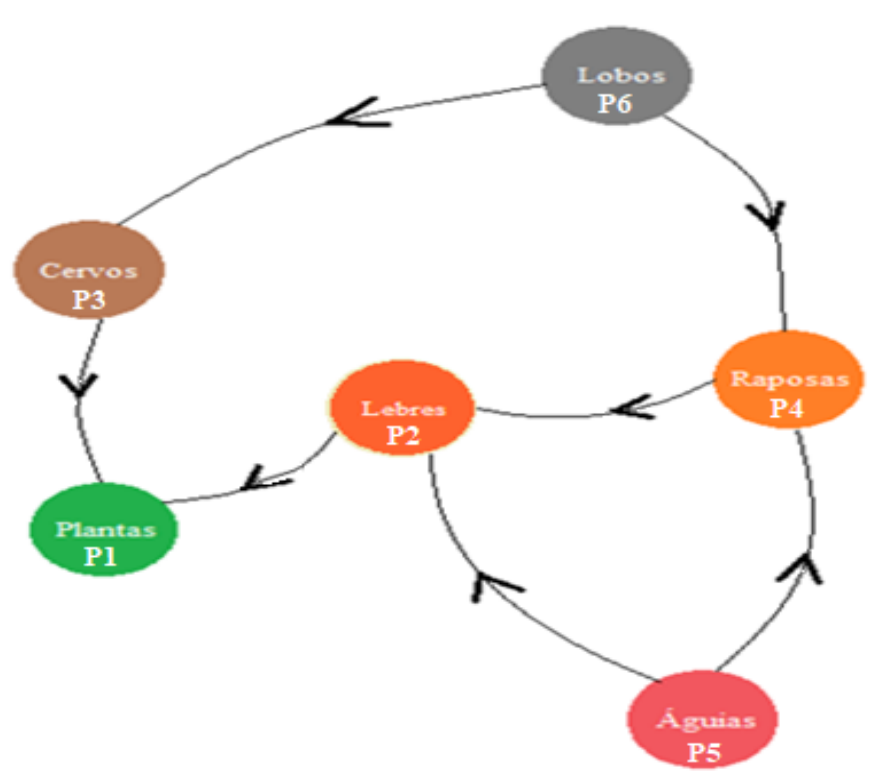

Cuja matriz é dada por:

$$
\mathrm{M}=\left[\begin{array}{llllll}
0 & 0 & 0 & 0 & 0 & 0 \\
1 & 0 & 0 & 0 & 0 & 0 \\
1 & 0 & 0 & 0 & 0 & 0 \\
0 & 1 & 0 & 0 & 0 & 0 \\
0 & 1 & 0 & 1 & 0 & 0 \\
0 & 0 & 1 & 1 & 0 & 0
\end{array}\right]
$$

Então é possível notar que nem a águia e nem o lobo são comidos por outro ser nesta teia alimentar, pois não há nenhuma conexão em suas colunas respectivas e no grafo eles não se ligam a ninguém, apenas recebem ligações.

E também é possível montar a matriz de 2 passos: 


$$
\mathrm{M}^{2}=\left[\begin{array}{llllll}
0 & 0 & 0 & 0 & 0 & 0 \\
0 & 0 & 0 & 0 & 0 & 0 \\
0 & 0 & 0 & 0 & 0 & 0 \\
1 & 0 & 0 & 0 & 0 & 0 \\
1 & 1 & 0 & 0 & 0 & 0 \\
1 & 1 & 0 & 0 & 0 & 0
\end{array}\right]
$$

Percebe-se que existem conexões que fazem com que os animais "maiores" se alimentem das plantas indiretamente. Tem-se então os seguintes caminhos de 2 passos:

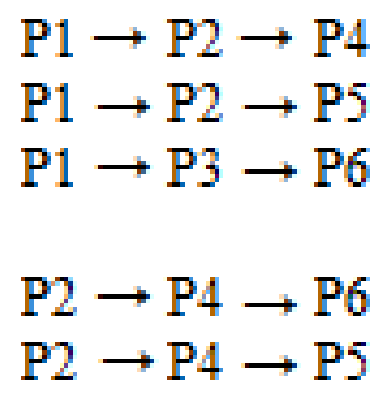

Então é possível observar que em uma teia alimentar existem relações indiretas entre os elementos que a compõe e que a falta de um componente pode afetar todos os níveis da cadeia existente, já que, apesar de não existir conexão direta, existem conexões de mais passos relacionando-os indiretamente.

\subsection{Torneios}

Os grafos dirigidos por dominância são, também, chamados de "torneios". Nestes casos, quando se tem $\mathrm{P}_{\mathrm{i}} \rightarrow$ $P_{j}$, isto quer dizer que $P_{i}$ venceu $P_{j}$, mas como $P_{j}$ não derrota $P_{i}$, é satisfeito o critério de dominância. (ANTON, H.; RORRES, C., 2012).

A soma das entradas na i-ésima linha da matriz $\mathrm{A}=\mathrm{M}+\mathrm{M}^{2}$ (esta soma será melhor analisada em 2.6) é o número total de conexões de 1 e 2 passos de $\mathrm{P}_{\mathrm{i}}$ para os outros vértices. A linha que tiver a maior soma identificará um vértice com conexões de 1 e 2 passos para todos os outros vértices de um grafo dirigido por dominância. (ANTON, H.; RORRES, C., 2012).

Exemplo: Oito amigos resolvem fazer uma pequena competição de xadrez entre eles. A competição se dará em três etapas eliminatórias, ou seja, quem perder sai da competição. Como xadrez é um jogo para dois, na primeira etapa terá 4 duplas, na segunda 2 e na última apenas 1, da qual sairá o vencedor. A seguir os resultados das etapas:

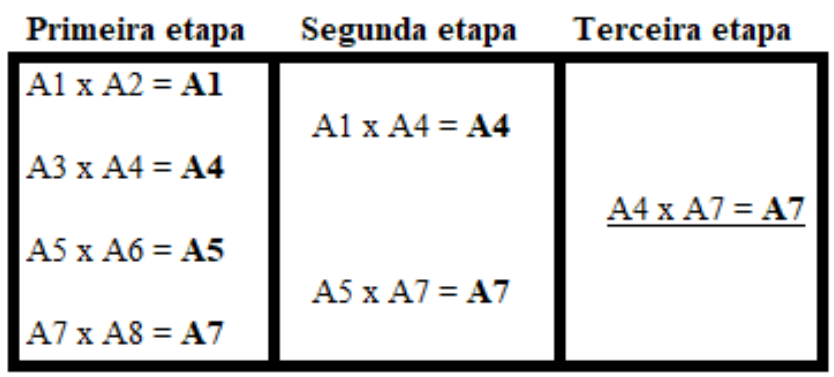


Através dos dados fornecidos, é possível encontrar o grafo desta competição:

Figura 3 - grafo relacionado ao exemplo anterior.

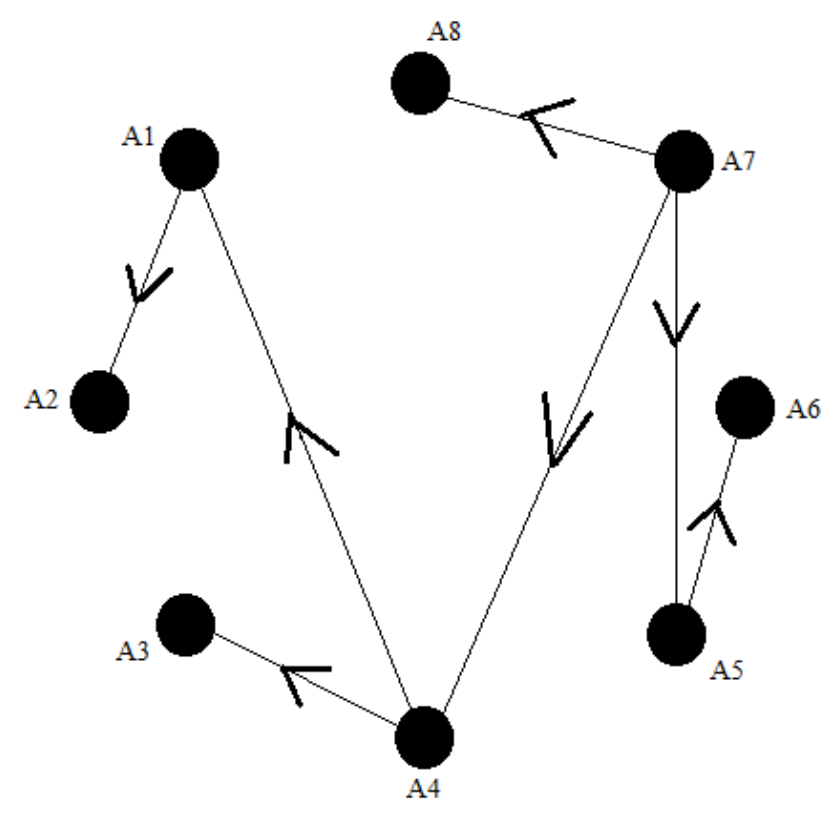

Fonte: própria autora

E também se pode obter as matrizes $\mathrm{M}$ e $\mathrm{M}^{2}$ :

$$
\begin{aligned}
\mathrm{M} & =\left[\begin{array}{llllllll}
0 & 1 & 0 & 0 & 0 & 0 & 0 & 0 \\
0 & 0 & 0 & 0 & 0 & 0 & 0 & 0 \\
0 & 0 & 0 & 0 & 0 & 0 & 0 & 0 \\
1 & 0 & 1 & 0 & 0 & 0 & 0 & 0 \\
0 & 0 & 0 & 0 & 0 & 1 & 0 & 0 \\
0 & 0 & 0 & 0 & 0 & 0 & 0 & 0 \\
0 & 0 & 0 & 1 & 1 & 0 & 0 & 1 \\
0 & 0 & 0 & 0 & 0 & 0 & 0 & 0
\end{array}\right] \\
\mathrm{M}^{2} & =\left[\begin{array}{llllllll}
0 & 0 & 0 & 0 & 0 & 0 & 0 & 0 \\
0 & 0 & 0 & 0 & 0 & 0 & 0 & 0 \\
0 & 0 & 0 & 0 & 0 & 0 & 0 & 0 \\
0 & 1 & 0 & 0 & 0 & 0 & 0 & 0 \\
0 & 0 & 0 & 0 & 0 & 0 & 0 & 0 \\
0 & 0 & 0 & 0 & 0 & 0 & 0 & 0 \\
1 & 0 & 1 & 0 & 0 & 1 & 0 & 0 \\
0 & 0 & 0 & 0 & 0 & 0 & 0 & 0
\end{array}\right]
\end{aligned}
$$


É possível concluir, com a análise do grafo e das matrizes, que o amigo A7 é o melhor enxadrista dentre todos, pois como ele ganha do A4, indiretamente ele ganha do A1 e do A2, além das vitórias que ele teve sobre o A8 e o A5, e ainda ele vence A6 indiretamente através do A5. O A4, apesar de ter ido até a última etapa como o A7, tem uma pontuação consideravelmente menor que o vencedor devido ao fato de que não tem como, com as rodadas acontecidas e os dados encontrados, comparar A4 com A5, A6 e A8. Então, não é possível dizer se ele ganharia ou perderia para um desses três, tudo que se sabe com estas informações é que es ses três perderam para o A7, para o qual A4 perdeu. Talvez com mais rodadas as pontuações fossem bem diferentes, mas com os dados fornecidos não se pode concluir isto. Observando " $\mathrm{A}=\mathrm{M}+\mathrm{M}^{2}$ " (esta dada em 2.6), encontra-se a pontuação obtida:

$$
\begin{aligned}
& \text { A1 }=1 \\
& \text { A2 }=0 \\
& \text { A3 }=0 \\
& \text { A4 }=3 \\
& \text { A5 }=1 \\
& \text { A6 }=0 \\
& \text { A7 }=6 \\
& \text { A8 }=0
\end{aligned}
$$

Observa-se que os quatro amigos que pontuaram 0 foram eliminados na primeira etapa, pois 0 significa que não venceu nenhuma partida. Os dois amigos que pontuaram 1 foram eliminados na segunda etapa, pois 1 significa que venceu uma partida. $\mathrm{O}$ amigo com 3 pontos, foi para a última etapa, porém perdeu, seus 3 pontos se devem a 2 vitórias diretas e uma indireta. $\mathrm{O}$ amigo com 6 pontos foi para a final e venceu, seus pontos indicam 3 vitórias diretas e 3 indiretas, tendo vencido quem derrotou o anterior.

Então, ao fazer o ranking de colocações, obtêm-se:

$$
\begin{gathered}
1^{\circ} \text { lugar: amigo A7 com } 6 \text { pontos } \\
2^{\circ} \text { lugar: amigo A4 com } 3 \text { pontos } \\
3^{\circ} \text { lugar: amigos A1 e A5 com } 1 \text { ponto } \\
4^{\circ} \text { lugar: amigos A2, A3, A6 e A8 sem pontos }
\end{gathered}
$$

\subsection{Poder de um vértice}

O poder de um vértice em um grafo dirigido por dominância é o número total de suas conexões de 1 e 2 passos para os outros vértices do grafo. De outro modo, o poder de um vértice $\mathrm{P}_{\mathrm{i}}$ é a soma das entradas da i-ésima linha da matriz $\mathrm{A}=\mathrm{M}+\mathrm{M}^{2}$. Assim, o vértice mais "poderoso" será aquele com o maior número de conexões de 1 ou 2 passos para os outros vértices do grafo, ou seja, aquele que se ligar a todos os outros vértices do grafo dirigido por dominância. (ANTON, H.; RORRES, C., 2012).

Exemplo: Considerando o exemplo da pequena competição de xadrez entre amigos, pode-se fazer o "poder" de cada amigo no final da competição.

Analisando por $\mathrm{A}=\mathrm{M}+\mathrm{M}^{2}$, é possível calcular o poder do amigo em questão, cada linha representando um deles. 


$$
\left[\begin{array}{llllllll}
0 & 1 & 0 & 0 & 0 & 0 & 0 & 0 \\
0 & 0 & 0 & 0 & 0 & 0 & 0 & 0 \\
0 & 0 & 0 & 0 & 0 & 0 & 0 & 0 \\
1 & 0 & 1 & 0 & 0 & 0 & 0 & 0 \\
0 & 0 & 0 & 0 & 0 & 1 & 0 & 0 \\
0 & 0 & 0 & 0 & 0 & 0 & 0 & 0 \\
0 & 0 & 0 & 1 & 1 & 0 & 0 & 1 \\
0 & 0 & 0 & 0 & 0 & 0 & 0 & 0
\end{array}\right]+\left[\begin{array}{llllllll}
0 & 0 & 0 & 0 & 0 & 0 & 0 & 0 \\
0 & 0 & 0 & 0 & 0 & 0 & 0 & 0 \\
0 & 0 & 0 & 0 & 0 & 0 & 0 & 0 \\
0 & 1 & 0 & 0 & 0 & 0 & 0 & 0 \\
0 & 0 & 0 & 0 & 0 & 0 & 0 & 0 \\
0 & 0 & 0 & 0 & 0 & 0 & 0 & 0 \\
1 & 0 & 1 & 0 & 0 & 1 & 0 & 0 \\
0 & 0 & 0 & 0 & 0 & 0 & 0 & 0
\end{array}\right] \begin{aligned}
& \mathrm{A} 1=1 \\
& \mathrm{~A} 2=0 \\
& \mathrm{~A} 3=0 \\
& \mathrm{~A} 4=3 \\
& \mathrm{~A} 5=1 \\
& \mathrm{~A} 6=0 \\
& \mathrm{~A} 7=6 \\
& \mathrm{~A} 8=0
\end{aligned}
$$

Então, realizando a soma das matrizes chega-se ao poder correspondente ao amigo analisado na linha. Dessa forma, conclui-se que o poder do amigo A7 é 6, o do A4 é 3, o dos amigos A1 e A5 é 1 e dos demais 0 , ou seja, poder nulo. Portanto o amigo A7 é o mais poderoso dentro da pequena competição de xadrez.

\section{Conclusões}

A teoria de grafos é importante no mundo da matemática, já que possibilita a "transformação" de situações cotidianas em problemas matemáticos, permitindo assim sua análise. A um grafo podem ser atribuídas diferentes matrizes, que representam diferentes etapas da análise do grafo. Portanto, através de tudo o que a teoria de grafos aborda, é possível observar detalhes de situações que não são reparados quando o problema não está neste formato. Com a teoria de grafos, pode-se quantizar as relações existentes em determinada situação, determinar o tipo, como relação mútua ou de dominância, direta, indireta e também é possível ver se existem atitudes isoladas dentro desta determinada situação.

\section{Referências}

KOLMAN, B.; HILL, D. Álgebra linear com aplicações 9 ed. Rio de Janeiro: LTC, 2014.

ANTON, H.; RORRES, C. Álgebra linear com aplicações 10 ed. Porto Alegre: Bookman, 2012.

COSTA, POLYANA POSSANI DA. Teoria de Grafos e suas Aplicações. 2011. Dissertação (Mestrado Profissional em Matemática Universitária) - Instituto de Geociências e Ciências Exatas, Universidade Estadual Paulista "Júlio de Mesquita Filho", Rio Claro.

FEOFILOFF, P.; KOHAYAKAWA, Y.; WAKABAYASHI, Y. Uma Introdução Sucinta à Teoria dos Grafos. São Paulo, 2011. 